\title{
The mathematical analysis for peristaltic flow of nano fluid in a curved channel with compliant walls
}

\author{
S. Nadeem • E. N. Maraj
}

Received: 19 September 2012/Accepted: 10 October 2012/Published online: 8 December 2012

(c) The Author(s) 2012. This article is published with open access at Springerlink.com

\begin{abstract}
In the present paper, we have investigated the peristaltic flow of nano fluid in a curved channel with compliant walls. The governing equations of nano fluid model for curved channel are derived including the effects of curvature. The highly nonlinear partial differential equations are simplified using the long wave length and low Reynolds number assumptions. The reduced nonlinear partial differential equation is solved analytically with the help of homotopy perturbation method. The physical features of pertinent parameters have been discussed by plotting the graphs of pressure rise, velocity, temperature, nano particle volume fraction and stream functions.
\end{abstract}

Keywords Peristaltic flow - Nano fluid .

Curved channel $\cdot$ Compliant walls

\section{Nomenclature}

$\bar{N} \quad$ Coordinate in cross stream

$\bar{S} \quad$ Coordinate in down stream

$\bar{Z} \quad$ Coordinate in vertical direction

$\bar{V} \quad$ Fluid velocity cross stream component

$\bar{U} \quad$ Fluid velocity down stream component

$R^{*} \quad$ Radius of curvature

$\lambda$ Wave length

$c \quad$ Wave speed

$b \quad$ Wave amplitude

Re Reynolds number

$\rho_{\mathrm{f}} \quad$ Fluid density

$\mu \quad$ Dynamic viscosity

S. Nadeem · E. N. Maraj $(\bowtie)$

Department of Mathematics, Quaid-i-Azam University, Islamabad, Pakistan

e-mail: ehnber@gmail.com $\left(\rho_{\mathrm{c}}\right)_{\mathrm{f}} \quad$ Heat capacity of the fluid

$\left(\rho_{\mathrm{c}}\right)_{\mathrm{p}} \quad$ Effective heat capacity of the nano particle material

$\kappa \quad$ Thermal conductivity

$D_{\mathrm{B}} \quad$ Brownian diffusion coefficient

$D_{\mathrm{T}} \quad$ Thermophoretic diffusion coefficient

$\sigma \quad$ Longitudinal tension per unit width

$m \quad$ Mass per unit area

C Coefficient of viscous damping

$\delta \quad$ Wave number

$k \quad$ Curvature parameter

$N_{\mathrm{b}} \quad$ Brownian motion parameter

$N_{\mathrm{t}} \quad$ Thermophoresis parameter

$G_{\mathrm{r}} \quad$ Local temperature Grashof number

$B_{\mathrm{r}} \quad$ Local nano particle Grashof number

\section{Introduction}

The topic of peristalsis is highly important in modern applied mathematics, engineering and physiological world. This is because of its many applications in real life such as swallowing food through the oesophagus, chyme motion in the gastrointestinal tract, in the vasomotion of small blood vessels such as venules, capillaries and arterioles, urine transport from kidney to bladder, in sanitary fluid transport, transport of corrosive fluids, a toxic liquid transport in the nuclear industry, etc,. Many researchers have discussed the peristaltic flows of viscous and non-Newtonian fluids theoretically and experimentally (Nadeem and Hameed 2007; Nadeem and Akbar 2009, 2010; Nadeem et al. 2010; Nadeem and Akram 2010a; Mekheimer 2008; Mekheimer and Abd elmabound 2008, 2011; Tripathi et al. 2010; Tripathi and Chhabra 1996). In these days, researchers have focussed their attention on the study of the nano fluid for 
different flow geometries. Recently, Nadeem and Akram (2010b) have considered the peristaltic flow of a nano fluid model in an asymmetric channel. In another study, Nadeem et al. (2012) have discussed the peristaltic flow of a nano fluid in an inclined asymmetric channel with partial slip and heat transfer. However, only few papers are available in literature, which discuss the peristaltic flow in a curved channel. Sato et al. (2000) have initiated the peristaltic flow in a curved channel. In their work Sato et al. concluded that the curvature parameter affects the fluid flow to a great extent. This effect is graphically shown in streamlines plots for different values of curvature parameter $k$. They concluded that the size of the bolus near the outer wall increases with increasing values of $k$, while the size of the bolus near the inner wall remains unaltered. However, when large values of Wessingber number $W_{\mathrm{e}}$ are taken in to the account the bolus near the inner wall disappears. Very recently, Hayat et al. (2011) have examined the peristaltic flow of viscous fluid in a curved channel with compliant walls. In this paper Hayat et al. concluded that the velocity decreases near the walls and increases near the centre of the channel when the compliant wall parameters are increased. Moreover, the curvature parameter effects are plotted for streamlines and size of the bolus grows with increase in curvature parameter.

The peristaltic flow of nano fluid model in curved channel is still not detonated. Therefore, in the present investigation we have highlighted the peristaltic flow of nano fluid in a curved symmetric channel. The governing equations of twodimensional nano fluid in a curved channel including the effects of curvature are modelled. After the non-dimensionalization and using assumptions of the long wave length and low Reynolds number approximation, the reduced highly nonlinear partial differential equation is solved analytically with the help of homotopy perturbation method. At the end the physical phenomena is discussed by plotting graphs.

\section{Mathematical formulation}

Consider a two-dimensional flow of an incompressible nano fluid in a curved channel of uniform thickness $2 a$. The channel walls are flexible and also considered as compliant on which small amplitude of the travelling waves are imposed. Defining $(\bar{N}, \bar{S}, \bar{Z})$ as the coordinates in the cross stream, down stream and vertical directions, respectively, and $R^{*}$ as the radius of curvature. The flow in the channel is induced by sinusoidal waves of small amplitude $b$ traveling along the flexible walls of the channel. The walls of the channel are considered as follows:

$\bar{N}=\eta=a+b \sin \left[\frac{2 \pi}{\lambda}(\bar{S}-c \bar{t})\right], \quad$ upper wall
$\bar{N}=-\eta=-a-b \sin \left[\frac{2 \pi}{\lambda}(\bar{S}-c \bar{t})\right] . \quad$ lower wall

In above equations $c$ is the wave speed and $\lambda$ denotes the wave length. Let $\bar{V}$ and $\bar{U}$ denote the velocity components in the cross stream and down stream directions, respectively. The governing equations of motion, energy and nano particle for curved channel are described as (Wang and Mujumdar 2007; Polidori et al. 2007)

$$
\begin{aligned}
& \frac{R^{*}}{\bar{N}+R^{*}} \frac{\partial}{\partial \bar{N}}\left(\frac{\left(\bar{N}+R^{*}\right)}{R^{*}} \bar{V}\right)+\frac{R^{*}}{\bar{N}+R^{*}} \frac{\partial \bar{U}}{\partial \bar{S}}=0, \\
& \rho_{\mathrm{f}}\left(\frac{\partial \bar{V}}{\partial \bar{t}}+\bar{V} \frac{\partial \bar{V}}{\partial \bar{N}}+\frac{R^{*} \bar{U}}{\bar{N}+R^{*}} \frac{\partial \bar{V}}{\partial \bar{S}}-\frac{\bar{U}^{2}}{\bar{N}+R^{*}}\right) \\
& =-\frac{\partial \bar{P}}{\partial \bar{N}}+\frac{2 \mu R^{*}}{\bar{N}+R^{*}} \frac{\partial}{\partial \bar{N}}\left(\left(\bar{N}+R^{*}\right) \frac{\partial \bar{V}}{\partial \bar{N}}\right) \\
& +\frac{\mu R^{*}}{\bar{N}+R^{*}} \frac{\partial}{\partial \bar{S}}\left(\frac{R^{*}}{\bar{N}+R^{*}} \frac{\partial \bar{V}}{\partial \bar{S}}+\frac{\partial \bar{U}}{\partial \bar{N}}-\frac{\bar{U}}{\bar{N}+R^{*}}\right) \\
& -\frac{2 \mu}{\bar{N}+R^{*}}\left(\frac{R^{*}}{\bar{N}+R^{*}} \frac{\partial \bar{U}}{\partial \bar{S}}+\frac{\bar{V}}{\bar{N}+R^{*}}\right), \\
& \rho_{\mathrm{f}}\left(\frac{\partial \bar{U}}{\partial \bar{t}}+\bar{V} \frac{\partial \bar{U}}{\partial \bar{N}}+\frac{R^{*} \bar{U}}{\bar{N}+R^{*}} \frac{\partial \bar{U}}{\partial \bar{S}}+\frac{\bar{U} \bar{V}}{\bar{N}+R^{*}}\right) \\
& =-\frac{R^{*}}{\bar{N}+R^{*}} \frac{\partial \bar{P}}{\partial \bar{S}}+\frac{\mu}{\bar{N}+R^{*}} \frac{\partial}{\partial \bar{N}} \\
& \times\left[\left(\bar{N}+R^{*}\right)\left(\frac{R^{*}}{\bar{N}+R^{*}} \frac{\partial \bar{V}}{\partial \bar{S}}+\frac{\partial \bar{U}}{\partial \bar{N}}-\frac{\bar{U}}{\bar{N}+R^{*}}\right)\right] \\
& +\frac{2 \mu R^{*}}{\bar{N}+R^{*}} \frac{\partial}{\partial \bar{S}}\left(\frac{R^{*}}{\bar{N}+R^{*}} \frac{\partial \bar{V}}{\partial \bar{S}}+\frac{\bar{V}}{\bar{N}+R^{*}}\right) \\
& +\rho g \alpha\left(\bar{T}-T_{0}\right)+\rho g \alpha\left(\bar{C}-C_{0}\right), \\
& \left(\rho_{\mathrm{c}}\right)_{\mathrm{f}} \frac{\mathrm{d} \bar{T}}{\mathrm{~d} \bar{t}}=\kappa \nabla^{2} \bar{T}+\left(\rho_{\mathrm{c}}\right)_{\mathrm{p}}\left[D_{\mathrm{B}} \nabla \bar{C} \cdot \nabla \bar{T}+\frac{D_{\mathrm{T}}}{T_{0}} \nabla \bar{T} \cdot \nabla \bar{T}\right],
\end{aligned}
$$

$\frac{\mathrm{d} \bar{C}}{\mathrm{~d} \bar{t}}=D_{\mathrm{B}} \nabla^{2} \bar{C}+\frac{D_{\mathrm{T}}}{T_{0}} \nabla^{2} \bar{T}$.

The corresponding boundary conditions for asymmetric channel having compliant walls are defined as

$\bar{U}=0$ at $\bar{S}= \pm \eta= \pm\left[a+b \sin \left(\frac{2 \pi}{\lambda}(\bar{S}-c \bar{t})\right)\right]$,

$$
\begin{aligned}
& \frac{R^{*}}{\bar{S}+R^{*}} \frac{\partial}{\partial \bar{S}} L(\eta)=\frac{R^{*}}{\bar{S}+R^{*}} \frac{\partial \bar{P}}{\partial \bar{S}}=\frac{\mu}{\bar{S}+R^{*}} \frac{\partial}{\partial \bar{S}} \\
& \quad \times\left[\left(\bar{S}+R^{*}\right)\left(\frac{R^{*}}{\bar{S}+R^{*}} \frac{\partial \bar{V}}{\partial \bar{N}}+\frac{\partial \bar{U}}{\partial \bar{S}}-\frac{\bar{U}}{\bar{S}+R^{*}}\right)\right] \\
& \quad+\frac{2 \mu R^{*}}{\bar{S}+R^{*}} \frac{\partial}{\partial \bar{N}}\left(\frac{R^{*}}{\bar{S}+R^{*}} \frac{\partial \bar{V}}{\partial \bar{N}}+\frac{\partial \bar{U}}{\partial \bar{S}}-\frac{\bar{U}}{\bar{S}+R^{*}}\right) \\
& -\rho_{\mathrm{f}}\left(\frac{\partial}{\partial t}+\bar{V} \frac{\partial \bar{U}}{\partial \bar{S}}+\frac{R^{*} \bar{U}}{\bar{S}+R^{*}} \frac{\partial \bar{U}}{\partial \bar{N}}+\frac{\bar{U} \bar{V}}{\bar{S}+R^{*}}\right) \text { at } \bar{S}= \pm \eta,
\end{aligned}
$$

$\bar{T}=T_{0}, \quad \bar{C}=C_{0}$ on $\bar{N}=-\eta$, 
$\bar{T}=T_{1}, \quad \bar{C}=C_{1}$ on $\bar{N}=\eta$.

Here $\rho_{\mathrm{f}}, \mu,\left(\rho_{\mathrm{c}}\right)_{\mathrm{f}},\left(\rho_{\mathrm{c}}\right)_{\mathrm{p}}, \kappa, D_{\mathrm{B}}$ and $D_{\mathrm{T}}$ are the fluid density, dynamic viscosity, heat capacity of the fluid, effective heat capacity of the nano particle material, Thermal conductivity, Brownian diffusion coefficient and thermophoretic diffusion coefficient, respectively. Moreover,

$L=-\sigma \frac{\partial^{2}}{\partial \bar{S}^{2}}+m \frac{\partial^{2}}{\partial t^{2}}+C \frac{\partial}{\partial t}$,

where $\sigma$ is the longitudinal tension per unit width, $m$ is the mass per unit area and $C$ is the coefficient of viscous damping.

Introducing the following non dimensional variables and velocity stream function relation

$n=\frac{\bar{N}}{\lambda}, s=\frac{\bar{S}}{a}, u=\frac{\bar{U}}{c}, v=\frac{\bar{V}}{c}, \psi=\frac{\bar{\psi}}{c a}, k=\frac{R^{*}}{a}, \operatorname{Re}=\frac{\rho_{\mathrm{f}} c a}{\mu}$,

$\delta=\frac{a}{\lambda}, p=\frac{a^{2} \bar{P}}{c \mu \lambda}, t=\frac{c \bar{t}}{\lambda}, \theta=\frac{\bar{T}-T_{0}}{T_{1}-T_{0}}, \sigma=\frac{\bar{C}-C_{0}}{C_{1}-C_{0}}$,

$G_{\mathrm{r}}=\frac{g \alpha a^{2}\left(T_{1}-T_{0}\right)}{v c}, \epsilon=\frac{b}{a}$,

$E_{1}=-\frac{\sigma a^{3}}{\lambda^{3} \mu c}, E_{2}=\frac{m c a^{3}}{\lambda^{3} \mu}, E_{3}=\frac{C a^{3}}{\lambda^{2} \mu}$,

$B_{\mathrm{r}}=\frac{g \alpha a^{2}\left(C_{1}-C_{0}\right)}{v c}, \alpha=\frac{\kappa}{\left(\rho_{\mathrm{c}}\right)_{\mathrm{f}}}, N_{\mathrm{b}}=\frac{\left(\rho_{\mathrm{c}}\right)_{\mathrm{p}} D_{\mathrm{B}} C_{0}}{\alpha\left(\rho_{\mathrm{c}}\right)_{\mathrm{f}}}$,

$N_{\mathrm{t}}=\frac{\left(\rho_{\mathrm{c}}\right)_{\mathrm{p}} D_{\mathrm{T}} T_{0}}{\alpha\left(\rho_{\mathrm{c}}\right)_{\mathrm{f}}}, u=-\frac{\partial \psi}{\partial r}, v=\delta \frac{k}{r+k} \frac{\partial \psi}{\partial x}$,

Equation (3) is identically satisfied and Eqs. (4)-(11) under long wavelength and low Reynolds number approximations are expressed in the following dimensionless form:

$\frac{\partial p}{\partial n}=0$

$\left(\frac{k}{n+k}\right) \frac{\partial p}{\partial s}=-\frac{1}{n+k} \frac{\partial}{\partial n}\left[\psi_{n n}+\frac{\left(1-\psi_{n}\right)}{n+k}\right]+G_{\mathrm{r}} \theta+B_{\mathrm{r}} \sigma$,

$\frac{\partial^{2} \theta}{\partial n^{2}}+\frac{1}{n+k} \frac{\partial \theta}{\partial n}+\left[N_{\mathrm{b}} \frac{\partial \sigma}{\partial n} \frac{\partial \theta}{\partial n}+N_{\mathrm{t}}\left(\frac{\partial \theta}{\partial n}\right)^{2}\right]=0$,

$\frac{\partial^{2} \sigma}{\partial n^{2}}+\frac{1}{n+k} \frac{\partial \sigma}{\partial n}+\frac{N_{\mathrm{t}}}{N_{\mathrm{b}}}\left[\frac{\partial^{2} \theta}{\partial n^{2}}+\frac{1}{n+k} \frac{\partial \theta}{\partial n}\right]=0$,

$\psi_{n}=0$ at $n= \pm \eta= \pm[1+\epsilon \sin 2 \pi(s-t)]$,

$-\frac{1}{n+k} \frac{\partial}{\partial n}\left[\psi_{n n}+\frac{\left(1-\psi_{n}\right)}{n+k}\right]+G_{\mathrm{r}} \theta+B_{\mathrm{r}} \sigma$

$=\frac{k}{n+k}\left[E_{1} \frac{\partial^{3} \eta}{\partial s^{3}}+E_{2} \frac{\partial^{3} \eta}{\partial t^{2} \partial s}+E_{1} \frac{\partial^{2} \eta}{\partial t \partial s}\right]$ at $n= \pm \eta$, $\theta=0, \quad \sigma=0$ at $n=-\eta$,

$\theta=1, \quad \sigma=1$ at $n=\eta$.

\section{Solution of the problem}

In order to solve coupled differential Eqs. (15) and (16) with the help of HPM, we construct the following equation (Abbasbandy 2006; He 2003; Wazwaz 2002):

$$
\begin{aligned}
& H(\theta, \widetilde{p})=(1-\widetilde{p}) \\
& \quad \times\left[\frac{\partial^{2} \theta}{\partial n^{2}}+\frac{1}{(n+k)} \frac{\partial \theta}{\partial n}-\left(\frac{\partial^{2} \widetilde{\theta}_{0}}{\partial n^{2}}+\frac{1}{(n+k)} \frac{\partial \widetilde{\theta}_{0}}{\partial n}\right)\right] \\
& +\widetilde{p}\left[\frac{\partial^{2} \theta}{\partial n^{2}}+\frac{1}{(n+k)} \frac{\partial \theta}{\partial n}+\left[N_{b} \frac{\partial \sigma}{\partial n} \frac{\partial \theta}{\partial n}+N_{t}\left(\frac{\partial \theta}{\partial n}\right)^{2}\right]=0,\right.
\end{aligned}
$$

$$
\begin{aligned}
H(\sigma, \widetilde{p})= & (1-\widetilde{p})\left[\frac{\partial^{2} \sigma}{\partial n^{2}}+\frac{1}{(n+k)} \frac{\partial \sigma}{\partial n}-\left(\frac{\partial^{2} \widetilde{\sigma}_{0}}{\partial n^{2}}+\frac{1}{(n+k)} \frac{\partial \widetilde{\sigma}_{0}}{\partial n}\right)\right] \\
& +\widetilde{p}\left[\frac{\partial^{2} \sigma}{\partial n^{2}}+\frac{1}{(n+k)} \frac{\partial \sigma}{\partial n}+\frac{N_{t}}{N_{b}}\left\{\frac{\partial^{2} \theta}{\partial n^{2}}+\frac{1}{n+k} \frac{\partial \theta}{\partial n}\right\}\right]=0 .
\end{aligned}
$$

Using

$\theta=\theta_{0}+\widetilde{p} \theta_{1}+\widetilde{p}^{2} \theta_{2}+\ldots$,

$\sigma=\sigma_{0}+\widetilde{p} \sigma_{1}+\widetilde{p}^{2} \sigma_{2}+\ldots$

With the help of Eqs. (23) and (24), equating the like powers of $\widetilde{p}$, we obtain the following systems:

\section{Zeroth-order system}

$\frac{\partial^{2} \theta_{0}}{\partial n^{2}}+\frac{1}{(n+k)} \frac{\partial \theta_{0}}{\partial n}=\frac{\partial^{2} \widetilde{\theta}_{0}}{\partial n^{2}}+\frac{1}{(n+k)} \frac{\partial \widetilde{\theta}_{0}}{\partial n}$,

$\frac{\partial^{2} \sigma_{0}}{\partial n^{2}}+\frac{1}{(n+k)} \frac{\partial \sigma_{0}}{\partial n}=\frac{\partial^{2} \widetilde{\sigma}_{0}}{\partial n^{2}}+\frac{1}{(n+k)} \frac{\partial \widetilde{\sigma}_{0}}{\partial n}$,

$\theta_{0}=0, \quad$ at $n=-\eta$

$\sigma_{0}=0, \quad$ at $n=-\eta$,

$\theta_{0}=1, \quad$ at $n=\eta$,

$\sigma_{0}=1, \quad$ at $n=\eta$.

\section{First-order system}

$$
\begin{gathered}
\frac{\partial^{2} \theta_{1}}{\partial n^{2}}+\frac{1}{(n+k)} \frac{\partial \theta_{1}}{\partial n}+\frac{\partial^{2} \theta_{0}}{\partial n^{2}}+\frac{1}{(n+k)} \frac{\partial \theta_{0}}{\partial n} \\
+\left[N_{\mathrm{b}} \frac{\partial \sigma_{0}}{\partial n} \frac{\partial \theta_{0}}{\partial n}+N_{\mathrm{t}}\left(\frac{\partial \theta_{0}}{\partial n}\right)^{2}\right]=0, \\
\frac{\partial^{2} \sigma_{1}}{\partial n^{2}}+\frac{1}{(n+k)} \frac{\partial \sigma_{1}}{\partial n}+\frac{\partial^{2} \sigma_{0}}{\partial n^{2}}+\frac{1}{(n+k)} \frac{\partial \sigma_{0}}{\partial n} \\
+\frac{N_{\mathrm{t}}}{N_{\mathrm{b}}}\left[\frac{\partial^{2} \theta_{0}}{\partial n^{2}}+\frac{1}{(n+k)} \frac{\partial \theta_{0}}{\partial n}\right]=0,
\end{gathered}
$$




$$
\begin{array}{lll}
\theta_{1}=0, & \text { at } n=-\eta, & \theta_{0}=\widetilde{\theta}_{0}=\frac{\log (k-\eta)-\log (k+n)}{\log (k-\eta)-\log (k+\eta)}, \\
\sigma_{1}=0, & \text { at } n=-\eta, & \\
\theta_{1}=0, & \text { at } n=\eta, & \sigma_{0}=\widetilde{\sigma}_{0}=\frac{\log (k-\eta)-\log (k+n)}{\log (k-\eta)-\log (k+\eta)}, \\
\sigma_{1}=0, & \text { at } n=\eta . &
\end{array}
$$

$$
\theta_{1}=\frac{2\left(N_{\mathrm{b}}+N_{\mathrm{t}}\right) \eta \log (k+n)}{\left(k^{2}-\eta^{2}\right)[\log (k+n)-\log (k+n)]^{3}}+\frac{\left(N_{\mathrm{b}}+N_{\mathrm{t}}\right)[(k-\eta)(n-\eta) \log (k-\eta)-(k+\eta)(n+\eta) \log (k+\eta)]}{(k+n)\left(k^{2}-\eta^{2}\right)[\log (k-\eta)-\log (k+\eta)]^{3}}
$$

\section{Second-order system}

$$
\begin{aligned}
& \frac{\partial^{2} \theta_{2}}{\partial n^{2}}+\frac{1}{(n+k)} \frac{\partial \theta_{2}}{\partial n}+N_{\mathrm{b}}\left(\frac{\partial \sigma_{1}}{\partial n} \frac{\partial \theta_{0}}{\partial n}+\frac{\partial \sigma_{0}}{\partial n} \frac{\partial \theta_{1}}{\partial n}\right) \\
& \quad+2 N_{\mathrm{t}}\left(\frac{\partial \theta_{0}}{\partial n} \frac{\partial \theta_{1}}{\partial n}\right)=0, \\
& \frac{\partial^{2} \sigma_{2}}{\partial n^{2}}+\frac{1}{(n+k)} \frac{\partial \sigma_{2}}{\partial n}+\frac{N_{\mathrm{t}}}{N_{\mathrm{b}}}\left[\frac{\partial^{2} \theta_{1}}{\partial n^{2}}+\frac{1}{(n+k)} \frac{\partial \theta_{1}}{\partial n}\right]=0, \\
& \theta_{2}=0, \quad \text { at } n=-\eta, \\
& \sigma_{2}=0, \quad \text { at } n=-\eta, \\
& \theta_{2}=0, \quad \text { at } n=\eta, \\
& \sigma_{2}=0, \quad \text { at } n=\eta .
\end{aligned}
$$

The solutions of the above systems satisfying the boundary conditions are directly written as

$$
\begin{aligned}
\sigma_{1}= & 0 \\
\theta_{2}= & -\frac{\left(N_{\mathrm{b}}+N_{\mathrm{t}}\right)\left(N_{\mathrm{b}}+2 N_{\mathrm{t}}\right)}{\left(k^{2}-\eta^{2}\right)^{2}[\log (k-\eta)-\log (k+\eta)]^{5}} \\
& \times[\eta \log (k+n)(4 \eta+k \log (k-\eta)-k \log (k+\eta)) . \\
& -\left\{\left((k-\eta)^{2}(n-\eta)(2 k+n+\eta) \log (k-\eta)^{2}\right.\right. \\
& +(k+\eta)(n+\eta) \log (k+\eta)(-8(k+n) \eta \\
& +(2 k+n+\eta)(k+\eta) \log (k+\eta))-2 \log (k-\eta) \\
& \times\left(-4(k+n)(k-\eta)(n-\eta) \eta+k^{2} n(2 k+n)\right. \\
& \left.\left.\left.\left.+\left(3 k^{2}+2 k n+n^{2}\right) \eta^{2}-\eta^{4}\right) \log (k+\eta)\right)\right\}\right]
\end{aligned}
$$

$$
\begin{aligned}
\theta(n, s)= & \frac{\log (k-\eta)-\log (k+n)}{\log (k-\eta)-\log (k+\eta)}+\frac{2\left(N_{\mathrm{b}}+N_{\mathrm{t}}\right) \eta \log (k+n)}{\left(k^{2}-\eta^{2}\right)[\log (k+n)-\log (k+n)]^{3}} \\
& +\frac{\left(N_{\mathrm{b}}+N_{\mathrm{t}}\right)[(k-\eta)(n-\eta) \log (k-\eta)-(k+\eta)(n+\eta) \log (k+\eta)]}{(k+n)\left(k^{2}-\eta^{2}\right)[\log (k-\eta)-\log (k+\eta)]^{3}} \\
& -\frac{\left(N_{\mathrm{b}}+N_{\mathrm{t}}\right)\left(N_{\mathrm{b}}+2 N_{\mathrm{t}}\right)}{\left(k^{2}-\eta^{2}\right)^{2}[\log (k-\eta)-\log (k+\eta)]^{5}}[\eta \log (k+n)(4 \eta+k \log (k-\eta)-k \log (k+\eta)) \\
& -\left\{(k-\eta)^{2}(n-\eta)(2 k+n+\eta) \log (k-\eta)^{2}+(k+\eta)(n+\eta) \log (k+\eta)\right. \\
& (-8(k+n) \eta+(2 k+n+\eta)(k+\eta) \log (k+\eta))-2 \log (k-\eta) \\
& \left.\left.\left.\left(-4(k+n)(k-\eta)(n-\eta) \eta+k^{2} n(2 k+n)+\left(3 k^{2}+2 k n+n^{2}\right) \eta^{2}-\eta^{4}\right) \log (k+\eta)\right)\right\}\right],
\end{aligned}
$$




$$
\begin{aligned}
\sigma(n, s)= & \frac{\log (k-\eta)-\log (k+n)}{\log (k-\eta)-\log (k+\eta)}-\frac{2 N_{\mathrm{t}}\left(N_{\mathrm{b}}+N_{\mathrm{t}}\right) \eta \log (k+n)}{N_{\mathrm{b}}\left(k^{2}-\eta^{2}\right)(\log (k-\eta)-\log (k+\eta))^{3}} \\
& -\frac{N_{\mathrm{t}}\left(N_{\mathrm{b}}+N_{\mathrm{t}}\right)((k-\eta)(n-\eta) \log (k-\eta)-(k+\eta)(n+\eta) \log (k+\eta))}{N_{\mathrm{b}}(k+n)\left(k^{2}-\eta^{2}\right)(\log (k-\eta)-\log (k+\eta))^{3}} .
\end{aligned}
$$

By using mathematical software Mathematica, the exact solution for the fluid velocity is evaluated after substituting expressions for $\theta$ and $\sigma$ in Eq. (14), which is discussed through graphs.

\section{Results and discussion}

The effect of various patient parameters, i.e. curvature parameter $k, N_{\mathrm{b}}, N_{\mathrm{t}}, B_{\mathrm{r}}$ and $G_{\mathrm{r}}$ are graphically discussed for

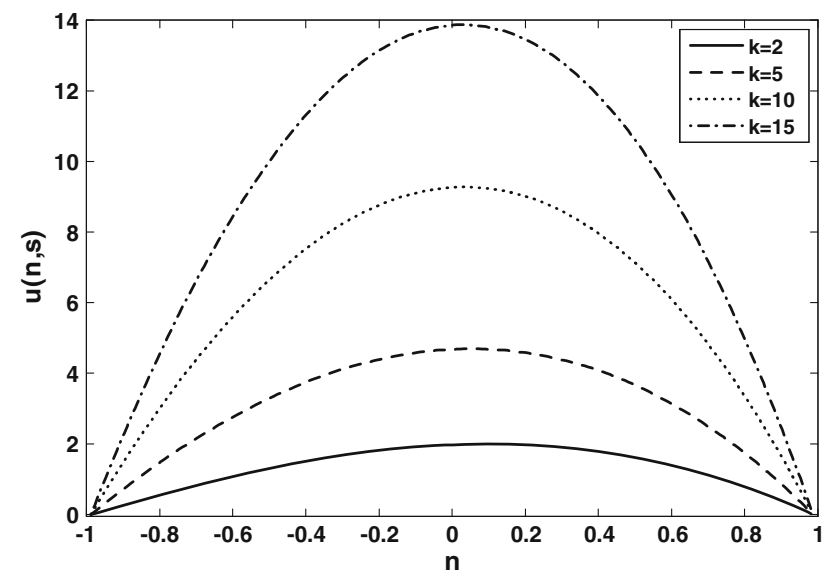

Fig. 1 The effect of curvature parameter $k$ on velocity profile. Plots are shown for different values of $k$ where $G_{\mathrm{r}}=0.2, B_{\mathrm{r}}=0.2$, $N_{\mathrm{t}}=0.2, N_{\mathrm{b}}=0.2, t=0.02, \epsilon=0.05, E_{1}=0.1, E_{2}=0.02, E_{3}=0.5$, $F=0.02$ and $s=0.01$

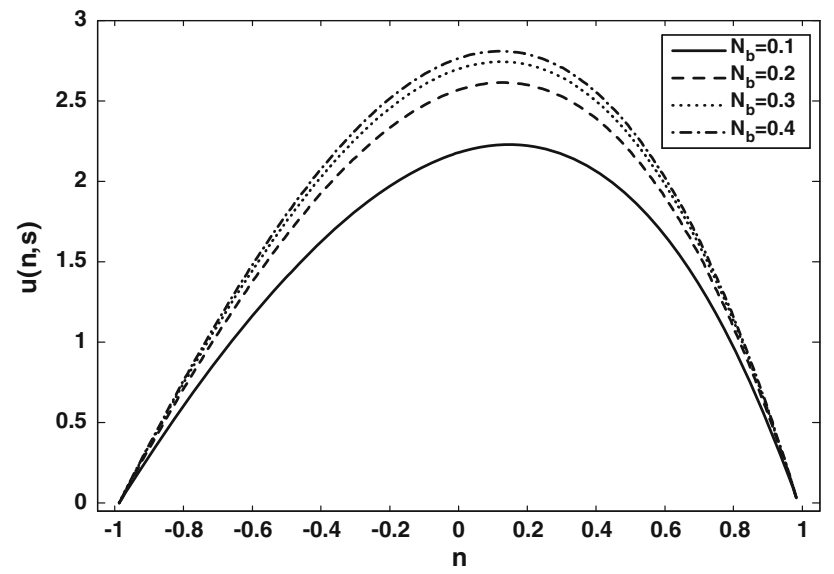

Fig. 2 The behaviour for different values of $N_{\mathrm{b}}$ where $G_{\mathrm{r}}=0.2$, $B_{\mathrm{r}}=1, N_{\mathrm{t}}=1.2, k=2.5, t=0.05, \epsilon=0.05, E_{1}=0.1, E_{2}=0.02$, $E_{3}=0.5, F=0.02$ and $s=0.01$ fluid velocity, temperature profile and concentration. Figures 1, 2, 3, 4 and 5 are plotted to illustrate the effects of pertinent parameters for velocity field. In Fig. 1, the effect of curvature parameter $k$ on fluid velocity is discussed. For small values of $k$, the velocity magnitude is small. The velocity increases with the increase in $k$. Maximum velocity is attained at the centre of the curved channel. Figure 2 displays the effect of Brownian motion parameter

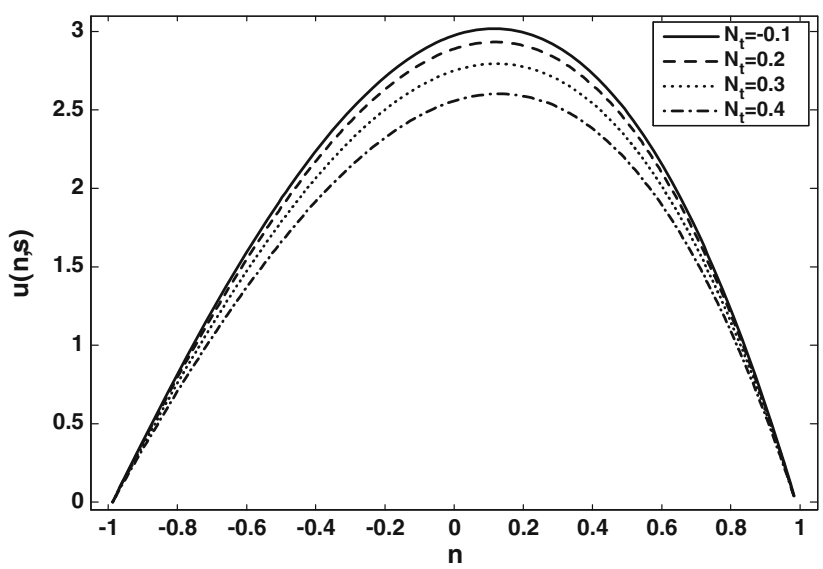

Fig. 3 A plot for velocity component $u(n, s)$ against $n$. It shows the effect of thermophoresis parameter $N_{\mathrm{t}}$ where $G_{\mathrm{r}}=0.2, B_{\mathrm{r}}=1$, $N_{\mathrm{b}}=0.02, k=2.5, t=0.05, \epsilon=0.05, E_{1}=0.1, E_{2}=0.02, E_{3}=0.5$, $F=0.02$ and $s=0.01$

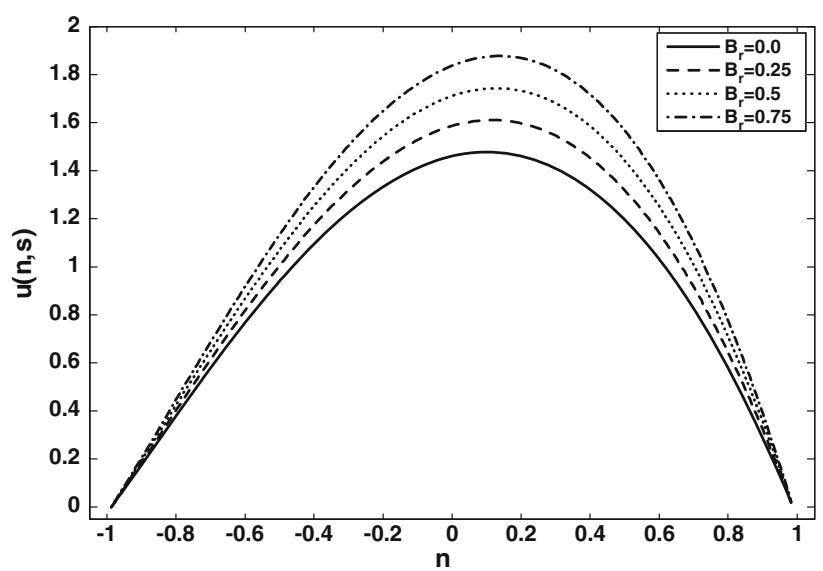

Fig. 4 The effect of $B_{\mathrm{r}}$ where $G_{\mathrm{r}}=0.2, N_{\mathrm{b}}=0.5, N_{\mathrm{t}}=0.2$, $k=1.5, t=0.05, \epsilon=0.05, E_{1}=0.1, E_{2}=0.02, E_{3}=0.5, F=0.02$ and $s=0.01$ 


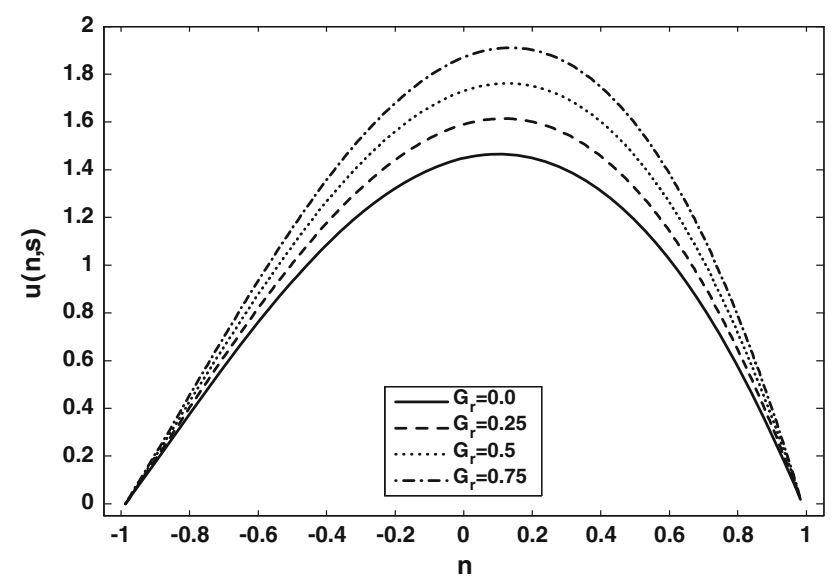

Fig. 5 The effect of $G_{\mathrm{r}}$ where $N_{\mathrm{b}}=0.5, B_{\mathrm{r}}=0.2, N_{\mathrm{t}}=0.2, k=$ $1.5, t=0.05, \epsilon=0.05, E_{1}=0.1, E_{2}=0.02, E_{3}=0.5, F=0.02$ and $s=0.01$

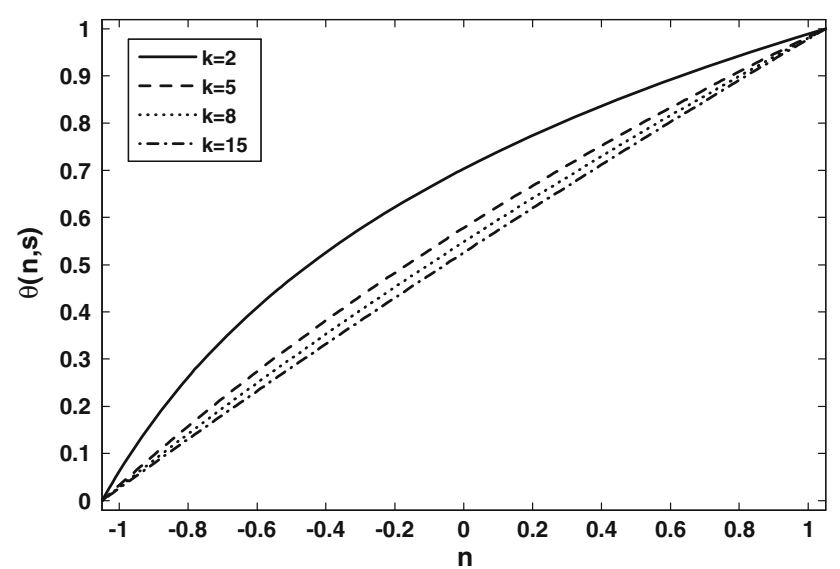

Fig. 6 A plot for temperature $\theta(n, s)$ against $n$. It shows the effects for different values of $k$ where $N_{\mathrm{t}}=0.5, N_{\mathrm{b}}=0.5, t=0.05, \epsilon=0.05$ and $s=0.3$

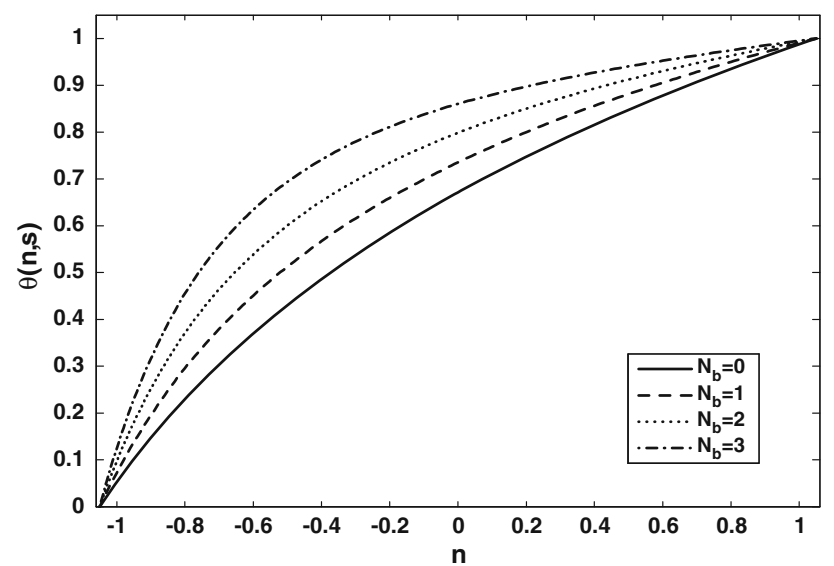

Fig. 7 The effect of $N_{\mathrm{b}}$ where $N_{\mathrm{t}}=0.5, k=2, t=0.05, \epsilon=0.05$ and $s=0.3$

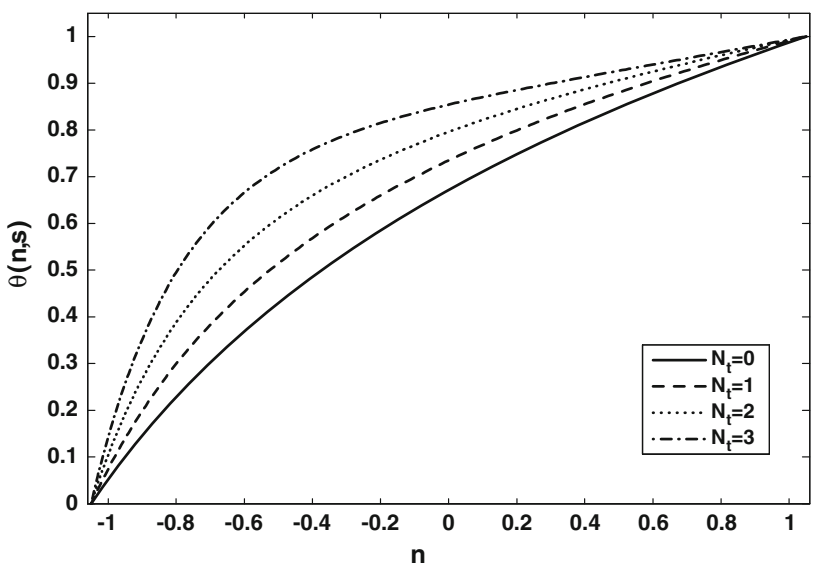

Fig. 8 The effect of $N_{\mathrm{t}}$ where $N_{\mathrm{b}}=0.5, k=2, t=0.05, \epsilon=0.05$ and $s=0.3$

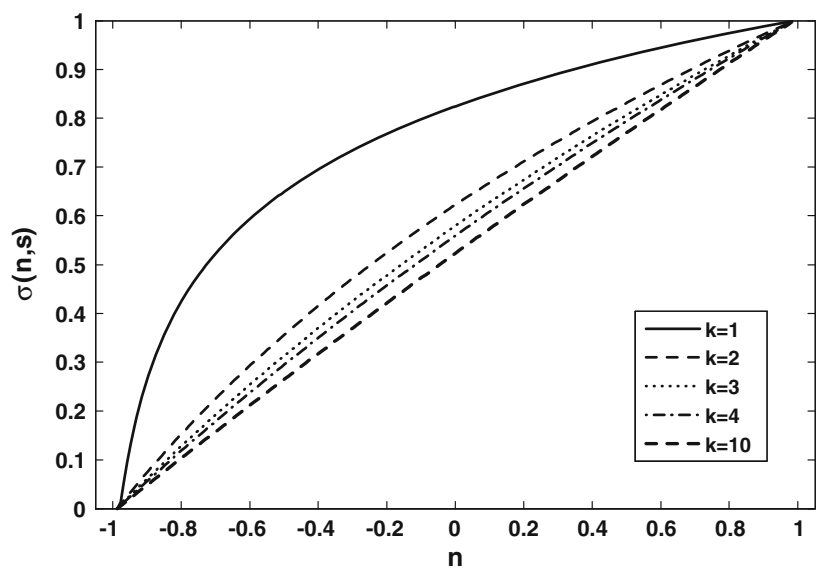

Fig. 9 Plot for $\sigma(n, s)$ against $n$. It shows the effect of $k$ where $N_{\mathrm{t}}=0.05, N_{\mathrm{b}}=0.05, t=0.05, \epsilon=0.05$ and $s=0.01$

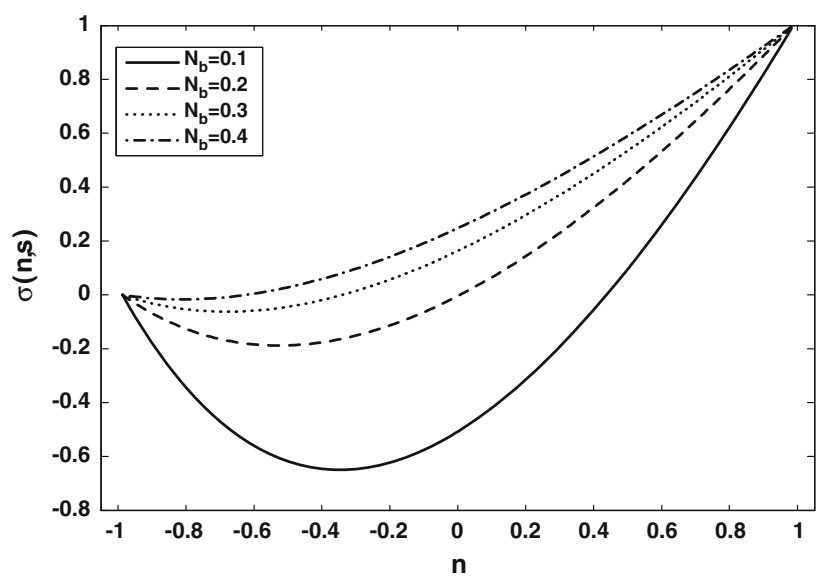

Fig. 10 The effect of $N_{\mathrm{b}}$ where $N_{\mathrm{t}}=2, k=5, t=0.05, \epsilon=0.05$ and $s=0.01$ 


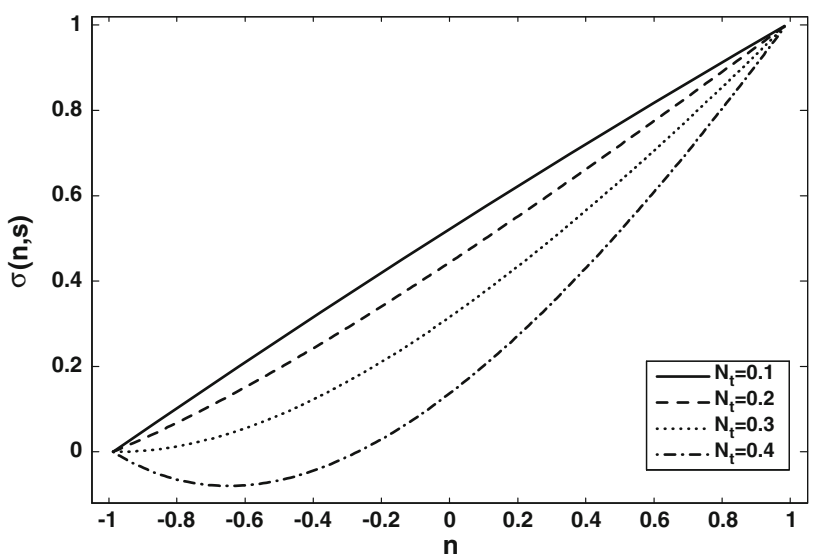

Fig. 11 The effect of $N_{\mathrm{t}}$ where $N_{\mathrm{b}}=0.01, k=5, t=0.05, \epsilon=0.05$ and $s=0.01$

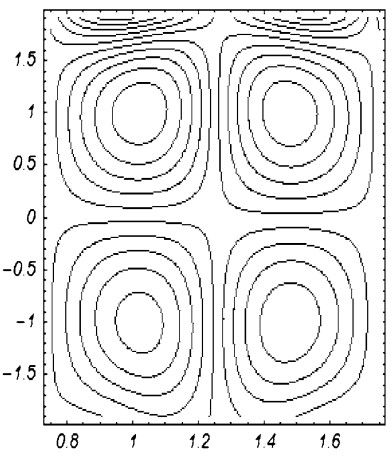

(a)

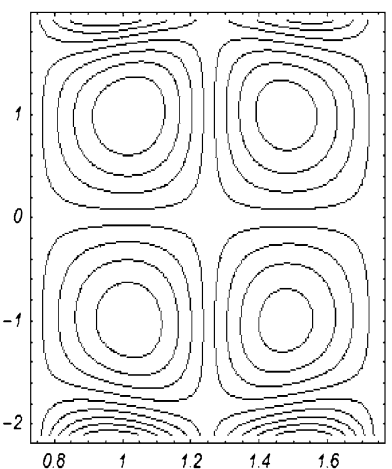

(c)

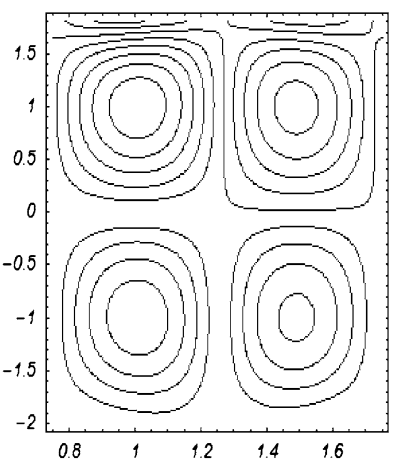

(b)
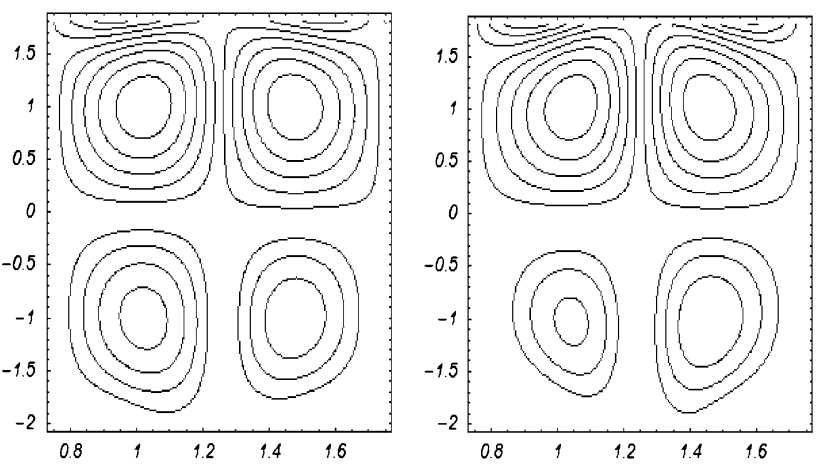

(d)

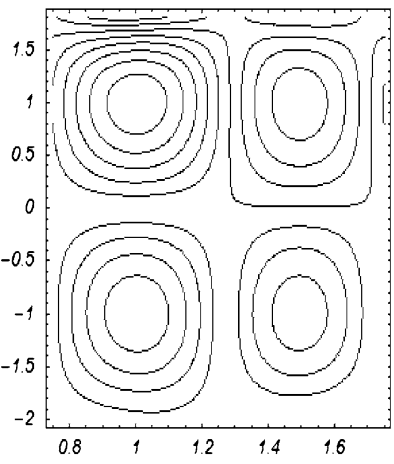

(a)
Fig. 13 The streamlines are plotted for against different values of $\epsilon$,i.e. $\mathbf{a} \epsilon=0.01, \mathbf{b} \epsilon=0.02, \mathbf{c} \epsilon=0.05, \mathbf{d} \epsilon=0.1$ while $B_{\mathrm{r}}=0.5$, $G_{\mathrm{r}}=0.5, N_{\mathrm{t}}=0.5, N_{\mathrm{b}}=2.5, t=0, k=2, E_{1}=1, E_{2}=0.5, E_{3}=$

velocity profile for different values of $B_{\mathrm{r}}$. The graphs are plotted for small values of $B_{\mathrm{r}}$; with the increase in $B_{\mathrm{r}}$, velocity increases. Figure 5 displays the graphs for different values of $G_{\mathrm{r}}$, and the fluid velocity increase with increase in $G_{\mathrm{r}}$. Figures 6,7 and 8 are graphs for temperature profile $\theta$ against $n$ for different values of $k, N_{\mathrm{b}}$ and $N_{\mathrm{t}}$. In Fig. 6, the effects of curvature parameter $k$ are discussed. We note a decrease in $\theta$ with an increase in curvature parameter $k$, i.e. the curvature parameter affects the temperature profile up to a large extent. For very large values of $k$, variation in $\theta$ gets small. Figure 7 is a graph for different values of $N_{\mathrm{b}}$, which shows increase in $\theta$ with the increase in $N_{\mathrm{b}}$. In Fig. 8 the effect of $N_{\mathrm{t}}$ is illustrated: increase in $N_{\mathrm{t}}$ increases $\theta$. Figures 9,10 and 11 are the plots for concentration $\sigma$ against $n$. Figure 9 shows the effect of curvature parameter $k$; we note that $\sigma$ decreases with the increase in $k$, but it remain almost steady for $k \geq 10$ onwards. Figure 10 shows the behaviour of $\sigma$ for different values of $N_{\mathrm{b}}$. It is observed that with increase in $N_{\mathrm{b}}, \sigma$ increases. The variation get small for large values of $N_{\mathrm{b}}$. Figure 11 shows the effect of $N_{\mathrm{t}}$ on $\sigma$. From Fig. 11 we conclude that $\sigma$ decreases with an increase in $N_{\mathrm{t}}$. The variation grows rapidly for large values of $N_{\mathrm{t}}$. In Fig. 12 streamlines are plotted for different values of $k$; here the
$N_{\mathrm{b}}$. Small variations in $N_{\mathrm{b}}$ are taken into the account. We observe that with the increase in $N_{\mathrm{b}}$, fluid velocity increases. The variation gets small for large values of $N_{\mathrm{b}}$. In Fig. 3 the effects of $N_{\mathrm{t}}$ are shown, and the effect of $N_{\mathrm{t}}$ is opposite to that of $N_{\mathrm{b}}$, i.e. fluid velocity decreases with the increase in $N_{\mathrm{t}}$. Figure 4 shows the graphical results of

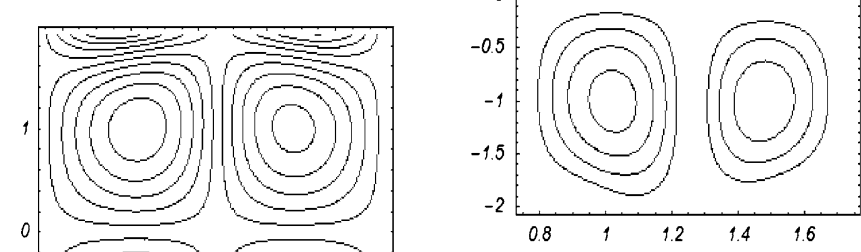

(c) 0.1 and $F=0.2$

(b)

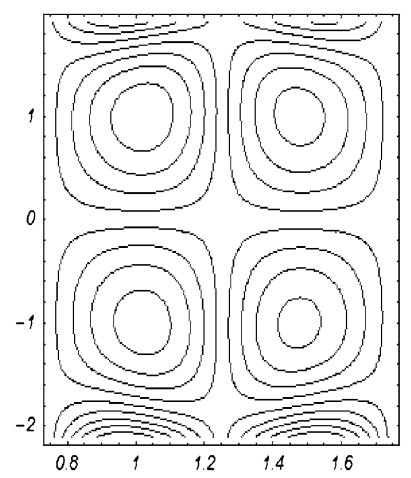

(d)
Fig. 12 The streamlines are plotted for against different values of curvature parameter $k$, i.e. a $k=2, \mathbf{b} k=4, \mathbf{c} k=6, \mathbf{d} k=8$ while $B_{\mathrm{r}}=0.5, G_{\mathrm{r}}=0.5, N_{\mathrm{t}}=0.5, N_{\mathrm{b}}=2.5, t=0, \epsilon=0.05, E_{1}=1, E_{2}=$ $0.5, E_{3}=0.1$ and $F=0.02$ 
size of the bolus decreases with the increase in $k$. The shape of the bolus near the upper half of the curved channel remains almost unaltered. However, near the lower half of the channel the shapes changes for different values of $k$. Figure 13 are the stream lines for different values of $\epsilon$ the shape of the bolus slightly changes from round to oval with the increase in values of $\epsilon$. We note a slight change in the shape of the bolus near the upper half of the curved channel. The size and the shape of the bolus changes for different values of $\epsilon$, near the lower half of the curved channel.

Open Access This article is distributed under the terms of the Creative Commons Attribution License which permits any use, distribution, and reproduction in any medium, provided the original author(s) and the source are credited.

\section{References}

Abbasbandy S (2006) Numerical solutions of integral equations: homotopy perturbation method and Adomian decomposition method. Appl Math Comp 173:493-500

Hayat T, Javed M, Hendi A (2011) Peristaltic transport of viscous fluid in a curved channel with compliant walls. Int J Heat Mass Transf 54:1615-1621

He J-H (2003) Homotopy perturbation method: a new non linear analytical technique. Appl Math Comput 135:73-79

Mekheimer KhS (2008) Effect of the induced magnetic field on peristaltic flow of a couple stress fluid. Phys Lett A 372: 4271-4278

Mekheimer KhS, Abd elmabound Y (2008) Influence of heat transfer and magnetic field on peristaltic transport of a Newtonian fluid in a vertical annulus: application of an endoscope. Phys Lett A 372:1657-1665

Mekheimer KhS, Abd elmabound Y (2011) Non-linear peristaltic transport of a second order fluid through porous medium. Appl Math Modell 35:2695-2710
Nadeem S, Hameed M (2007) Unsteady MHD flow of a nonNewtonian fluid on a porous plate. J Math Anal Appl 325: 724-733

Nadeem S, Akbar NS (2009) Effects of heat transfer on the peristaltic transport of MHD Newtonian fluid with variable viscosity: application of Adomian decomposition method. Commun Nonlinear Sci Numer Simul 14:3844-3855

Nadeem S, Akbar NS (2010) Influence of temperature dependent viscosity on peristaltic transport of a Newtonian fluid: application of an endoscope. Appl Math Comput 216:3606-3619

Nadeem S, Akbar NS, Hayat T, Obaidat S (2012) Peristaltic flow of a Williamson fluid in an inclined asymmetric channel with partial slip and heat transfer. Int J Heat Mass Transf 55:1855-1862

Nadeem S, Akbar NS, Bibi N, Ashiq S (2010) Influence of heat and mass transfer on peristaltic flow of a third order fluid in a diverging tube. Commun Nonlinear Sci Numer Simul 15:29162931

Nadeem S, Akram S (2010a) Heat transfer in peristaltic flow of MHD fluid with partial slip. Commun Nonlinear Sci Numer Simul 15:312-321

Nadeem S, Akram S (2010b) Peristaltic flow of a Williamson fluid in an asymmetric channel. Commun Nonlinear Sci Numer Simul 615:1705-1716

Polidori G, Fohanno S, Nguyen CT (2007) A note on heat transfer modelling of Newtonian nanofluids in laminar free convection. Int J Therm Sci 46:739-744

Sato H, Kawai T, Fujita T, Okabe M (2000) Two dimensional peristaltic flow in curved channels. Trans Jpn Soc Mech Eng B 66:679-685

Tripathi A, Chhabra RP (1996) Transverse laminar flow of nonNewtonian fluid over a bank of long cylinders. Chem Engg Comm 147:197-212

Tripathi D, Pendey KS, Das S (2010) Peristaltic flow of viscoelastic fluid with fractional maxwell model through a channel. Appl Math Comput 215:3645-3654

Wang X-Q, Mujumdar AS (2007) Heat transfer characteristics of nanofluids: a review. Int J Therm Sci 46:1-19

Wazwaz AM (2002) A new method for solving singular initial value problems in the second order ordinary differential equations. Appl Math Comput 128:45-57 